same species provokes the production of both humoral and cell-bound antibody; but the former generally does not destroy the graft. Transplantation immunity thus possesses an important feature in common with delayed hypersensitivity. Further similarities arise from the routes of immunization used - the intravenous injection of cells provokes only a poor response in the rabbit-as well as from the tempo of the full transplantation immunity reaction which develops before the production of humoral antibody reaches its maximum. More significantly, the cellular infiltration of homografts resembles the granuloma induced by tubercle bacillary wax. The nature of the cell first stimulated by the antigen may determine the type of the response, so that a single antigen may, in different circumstances, elicit either a humoral or a cell-bound antibody. Alternatively, tissue cells may be supposed to possess isoantigens of different kinds each responsible for one kind of antibody.

The possibility that a single molecular species of antigen can provoke simultaneously both delayed and immediate sensitivity to different determinant groups on it was discussed by P. G. H. Gell and B. Benacerraf. In a study of various types of immunological reaction to proteins conjugated with such active haptenes as picryl chloride, they have demonstrated a dissociation between immediate and delayed skin reactivity to the same antigen. The absence of recognizable antibodies to the protein carriers used in these conjugates, at a time when their intradermal injection proved capable of exciting a delayed skin reaction, confirms the view that reactions of this type do not depend on conventional antibodies in the circulation : at this time, antibodies were present specific to the haptenic group. Under other conditions, delayed sensitivity to the haptenic group was also demonstrable. They questioned the view that the state of delayed hypersensitivity can be regarded as an early, perhaps immature, stage of immunity. Rather, they felt that it should be considered as a distinctive response to certain quali. tatively different, possibly less dominant, groups on the antigenic molecule.

With the recognition of the close participation of leucocytes in the transference of specific delayed hypersensitivity, J. L. Gowans's account of the lifehistory of lymphocytes acquires particular relevance. Experiments on rats have shown that the output of these cells from the thoracic duct is sufficient to replace all the lymphocytes in the blood many times daily; the production of new small lymphoeytes is much lower and their survival time much longer than was formerly supposed. These cells, moreover, appear to circulate freely through the tissue spaces and in this extravascular transit they may be the effector cells in immunological reactions of the delayed type.

The features of the immediate and delayed 'tuberculin-type' reactions to trichophytin in guinea pigs that can be evoked either after an infection or an inoculation with the killed mycelium were described by C. N. Cruickshank, M. D. Trotter and M. R. Wood. They found that these responses were associated with a transferable passive cutaneous anaphylaxis, but that they could occur in the absence of any detectable precipitating antibodies. Chemical fractionation of the mycelium showed that the antigenic material was mainly a polysaccharide containing equal proportions of glucose and mannose. Finally, R. M. Gordon and M. Lavoipierre, in discussing immediate and delayed reactions to insect bites, pointed out that in certain instances the late reactions ordinarily attributed to the saliva of the vector may be confused with that caused by some parasite introduced into the tissues at the time.

\title{
THE ELECTRIC ARC IN WELDING
}

$\mathrm{F}^{\circ}$ OLLOWING the practice of the previous two years, a third "Joining of Metals" Conference was held at the University of Birmingham on June 25 under the chairmanship of Prof. E. C. Rollason, head of the Department of Industrial Metallurgy. The subject on this occasion was "The Electric Arc in Welding"; five papers were presented, and the conference was attended by about one hundred representatives of industry, the research associations and the universities. Prof. Rollason explained that the purpose of these conferences was to further the development and teaching of the basic processes underlying the practice of metal joining, and pointed out that, in arc welding in particular, much less effort had been directed towards fundamentals than to the empirical development of modern are welding processes. $\mathrm{He}_{\theta}$ then suggested that there were three ways in which the arc interacted with the metal which were of significance in welding. First, there was heat transfer from the arc which was responsible for the formation of the weld pool; secondly, the chemico-metallurgical reactions taking place between the high-temperature gases in the arc atmosphere and the weld metal; and thirdly, the transfer of metal droplets across the arc which can take place against gravity and for which no satisfactory mechanism had yet been advanced.

Mr. D. R. Milner, of the Department of Industrial Metallurgy, then surveyed the present state of knowledge of those aspects of arc physics which were pertinent to these problems. Throughout the main body of the arc electrical energy is utilized to heat the gas to such a temperature that it becomes thermally ionized and is thus able to provide the electrons and ions necessary to carry the required current. At the anode the electron stream, and at the cathode the positive ion stream, provide the source of energy for melting the metal. For low-current ares the anode processes are reasonably well established, but less is known of conditions at the cathode. However, for high-current welding systems little information is available in either case. Heat and mass transfer from the arc column to the electrodes, which determ. ines the reactions occurring between the arc atmosphere and the weld motal and controls the rate at which they take place, is dependent upon the energy dissipated, the gas properties and the mode of heat transfer. Of particular interest in this respect are plasma-jets, which Maecker has demonstrated exist wherever there is a constriction in the arc, such as a 
cathode spot, giving rise to gas velocities of the order of $10^{4}$ to $10^{5} \mathrm{~cm}$. per sec.

Dr. G. R. Salter, of the Department of Industrial Metallurgy, contributed a paper which described the results of an investigation of the absorption of oxygen by titanium melted by an electric arc in an atmosphere of argon containing controlled quantities of oxygen. The effect of time, oxygen partial pressure, arc length, current, electrode composition and gas flow conditions had been determined. The interpretation of the results led to the conclusion that in this system the rate-controlling process was the diffusion of oxygen across a 'stagnant' boundary-layer of gas of the order of $10^{-3} \mathrm{~cm}$. thickness, adjacent to the molten metal, which took place over a high temperature active area where the oxygen was dissociated. The magnitude of the active area was determined by the current and are length, and the thickness of the boundary layer by the velocity of the cathode plasma-jet which impinged on the anode.

Mr. J. B. Wilkinson, also of the Department of Industrial Metallurgy, gave an account of work on heat transfer in which energy balances had been determined for arcs operating between a tungsten cathode and a water-cooled copper anode in atmospheres of argon, nitrogen, helium and hydrogen. The existence of plasma-jets in these arcs had been demonstrated, their velocities estimated and attempts made to separate the heat transfer from the plasmajet from that due to electron heating of the anode. The interpretation of the measurements of the heat transferred from the plasma-jet was along similar lines to that proposed by Salter for mass transfer, that is to say, with convection transferring the heat to a boundary-layer adjacent to the metal surface. Some success had been achieved by the application of a conventional non-dimensional treatment of convective heat transfer with the plasma-jet replaced by an equivalent source of hot gas emerging from a tube.

In addition to their role in heat and mass transfer, plasma-jets are also responsible for the transfer of metal droplets from the molten wire electrode to the weld plate. This was shown by Mr. J. C. Needham, who described work carried out at the Electrical Research Association in which a study had been made of the detachment and flight of aluminium droplets by high-speed colour photography $(8,000$ frames per sec.). The existence and effect of the plasma-jet could be inferred from a stream of metal vapour emanating from the droplets flowing in the direction of the jet and from the fact that the velocity of the drops continued to increase, with accelerations of $10 g$ to $100 \mathrm{~g}$, after they had been detached from the electrode wire, attaining terminal velocities in excess of $500 \mathrm{~cm} . / \mathrm{sec}$. Experimental determinations of the droplet velocity as a function of current, derived from the photographs taken by Needham, and from trajectory determinations by Mr. C. J. Cooksey of the University of Birmingham, compared well with theoretical calculations based on a model in which the drop became detached when the force exerted on it by the plasma-jet exceeded the restraining force of surface tension, and was then freely accelerated across the are by the impinging gas stream.

An interesting characteristic of the electric arc is that if it is intensively cooled, for example, by operating it through a narrow cooled orifice, then the core temperature is increased. This is because the con. ducting area contracts, so that which remains must have a higher degree of ionization and hence a higher temperature in order to maintain the required eurrent. Spectroscopists and arc physicists have utilized this property of the are to heat gas to temperatures up to $50,000^{\circ} \mathrm{C}$. for the measurement of collision cross-sections and the transition probabilities of ionized and excited atoms and for fundamental magnetohydrodynamic studies. Mr. A. R. Moss, of the Ministry of Supply, elaborated on the behaviour of the constricted arc and showed how it could be harnessed to technological advantage. $\mathrm{He}$ described the various types of plasma-jet projectors and constricted arc torches developed in the Armament Research and Development Establishment, with emphasis on the design and characteristics of devices operating with a power consumption up to $100 \mathrm{kV}$. amp., although much more powerful equipments were mentioned. Their many potential technological applications include the melting, cutting and spraying of metallic and non-metallic materials in noncontaminating atmospheres, chemical synthesis and the production of high-temperature gas streams at hypersonic velocities. $\quad$ D. R. MILNER

\section{INTERNATIONAL CONGRESS ON ACOUSTICS}

$\mathrm{T}$ HE third International Congress on Acoustics was held in Stuttgart during September 1--8 under the presidency of Prof. Erwin Meyer.

The first of the series was held in Delft in 1953 and the second in Cambridge, Massachusetts, in 1956. Already the number of participants has risen from the original 600 to more than 1,000, and in the recent congress necessitated the individual papers being read in eight concurrent sessions. The mornings were devoted to review lectures, which all could attend. These, and the papers presented, covered architectural acousties, ultrasonic techniques and their applications to the study of molecular physics, acoustical properties of materials employed in industry, noise and its abatement (in particular aircraft noise), physiological and psychological acoustics.
If one tries to assess the progress made since the last congress and which is likely to continue in the future, one would perhaps select the last two of these as of most interest. The two reviews given on the subject of the noise of jets and the quantity of smaller papers which followed illustrated the strides which have been made since the last congress, where the subject of aeronauties interested but a few speakers. The increasing noise-level to which we are subjected has stimulated more research into the functioning of the ear and the human processes of sound perception, which also brought out a stimulating array of papers.

In building acoustics and molecular acoustics one has the feeling that the peak of development has passed. In the former subject it seems now to be a 